\title{
Invalid inpatient stays and their potential reimbursement
}

\author{
I Garcia Lema ${ }^{1,2^{*}}$, T Silva-Costa ${ }^{1,2}$, A Freitas ${ }^{1,2}$ \\ From 26th Patient Classification Systems International (PCSI) Working Conference \\ Munich, Germany. 15-18 September 2010
}

\section{Introduction}

An inpatient is usually defined as a patient who is admitted to a hospital or clinic for treatment that requires at least one overnight stay. However, since 1 August 2006, and for reimbursement purposes, under Portuguese law the length of stay must be longer than 24 hours to be considered a valid inpatient stay.

The Portuguese National Health System hospitals are financed through two different schemes. For patients with a third party responsible for health expenditure, the hospital charges a fixed price by DRG for each inpatient stay, and for some outpatient visits such as chemotherapy, ambulatory surgery, and dialysis, among others.

For patients covered solely by the National Health System, hospitals are financed through an annual agreement. Under this agreement, there is a single fixed price for inpatient stays as well as those outpatient visits which have a DRG associated. Each type of production is valued by applying that price, adjusted by the casemix index for that type, to the amount of equivalent inpatient stays (adjusted to the medium length of stay outside the considered normality limits) or outpatient visits.

Stays under 24 hours, since they are registered as inpatient stays, are not eligible for inpatient reimbursement under either scheme. In addition, they are also not considered proper outpatient visits, and are not paid as such to the hospital. Even so, the hospital incurs costs with these patients, often including surgical procedures or other costly treatments. Our aim is to show how invalid inpatient stays affect both the hospital's budget and funding.

${ }^{1}$ Serviço de Bioestatística e Informática Médica, Faculdade de Medicina da Universidade do Porto, Porto, Portugal

Full list of author information is available at the end of the article

\section{Methods}

We identified the invalid inpatient stays, both for medical and surgical DRG, registered in 2008 in all public hospitals under the north region health administration (16 hospitals). We then proceeded to calculate their weight on the total number of inpatients for each hospital, the value the hospital would receive if those stays were registered as outpatient visits (both for all patients having a third party financial responsible and for all patients being covered by the National Health Service), and the impact of this latter value, as a percentage, on the hospital outpatient budget of the annual agreement.

\section{Results}

Overall we identified 2,907 stays representing $0.90 \%$ of all inpatient stays. Having no direct knowledge of the costs involved in the treatment of these patients, and using their ambulatory price for DRG as a proxy, we may state that, to the hospitals, these stays imply an expenditure that amounts to $€ 1,109,903.98$, for which they will not be reimbursed.

The prevalence of these cases varied from hospital to hospital, ranging from $0.10 \%$ to $2.40 \%$ of the total of inpatients (as shown in table 1). The registry of these stays as outpatient visits, assuming all patients were covered by the National Health Service, would allow hospitals to receive further funding of $€ 878,875$.57. In individual hospitals, that funding would represent from $0.04 \%$ up to $3.85 \%$ of the allotted budget for ambulatory cases classified by DRG.

The differences between hospitals are not explained by the type of hospital, as defined by the Central Administration of the Health System for funding purposes. Hospitals $\mathrm{M}$ and $\mathrm{A}$, for instance, belong to the same economic group but show clearly different admission policies. In hospital $M$, the proportional gain of reconsidering these admissions is just $0.19 \%$, corresponding to 
Table 1 Invalid Inpatients (length of stay under 24 hours), 2008

\begin{tabular}{llllll}
\hline Hospitals & $\mathbf{N}$ & \% & Reimbursable Value Not-NHS & Reimbursable Value NHS & Weight on Budget (NHS) \\
\hline A & 263 & 1.10 & $€ 135,776.17$ & $€ 97,547.03$ & $3.85 \%$ \\
B & 611 & 2.40 & $€ 251,163.42$ & $€ 177,136.62$ & $3.77 \%$ \\
C & 447 & 1.50 & $€ 179,499.43$ & $€ 185,296.26$ & $2.73 \%$ \\
D & 93 & 0.60 & $€ 21,927.06$ & $€ 13,187.26$ & $2.28 \%$ \\
E & 349 & 1.40 & $€ 155,451.66$ & $€ 114,649.19$ & $1.64 \%$ \\
F & 27 & 0.30 & $€ 6,593.53$ & $€ 11,291.34$ & $0.78 \%$ \\
G & 478 & 1.40 & $€ 124,546.82$ & $€ 122,639.12$ & $0.75 \%$ \\
H & 107 & 0.70 & $€ 42,635.22$ & $€ 23,017.46$ & $0.69 \%$ \\
I & 193 & 0.90 & $€ 39,721.42$ & $€ 30,214.05$ & $0.48 \%$ \\
$J$ & 12 & 0.70 & $€ 4,525.08$ & $€ 2,917.73$ & $0.32 \%$ \\
K & 18 & 0.10 & $€ 13,561.40$ & $€ 8,598.14$ & $0.27 \%$ \\
L & 64 & 0.30 & $€ 8,090.17$ & $€ 9,129.82$ & $0.20 \%$ \\
M & 99 & 0.20 & $€ 46,775.88$ & $€ 41,266.54$ & $0.19 \%$ \\
N & 34 & 0.70 & $€ 4,070.98$ & $€ 1,913.86$ & $0.17 \%$ \\
O & 100 & 0.90 & $€ 67,770.03$ & $€ 33,047.41$ & $0.09 \%$ \\
P & 12 & 0.10 & $€ 7,895.70$ & $€ 6,969.74$ & $0.04 \%$ \\
Total & 2,907 & 0.90 & $€ 1,109,903.98$ & $€ 878,875.57$ & $0.66 \%$ \\
\hline
\end{tabular}

$€ 41,266.54$; whereas, in hospital $\mathrm{A}$, this gain would represent $3.85 \%$, corresponding to $€ 97,547.03$.

\section{Conclusions}

This short paper demonstrates that a proper management of patient admission may increase hospital funding. Invalid inpatient stays result in no kind of reimbursement for the hospital. The timely detection of these cases, and the changing of their registry to outpatient visits, would allow the hospital to seek reimbursement for the costs it has incurred with these patients.

\section{Author details}

'Serviço de Bioestatística e Informática Médica, Faculdade de Medicina da Universidade do Porto, Porto, Portugal. ${ }^{2}$ CINTESIS - Center for Research on Health Technologies and Information Systems, Porto, Portugal.

Published: 6 October 2010

doi:10.1186/1472-6963-10-S2-A13

Cite this article as: Lema et al:: Invalid inpatient stays and their potential reimbursement. BMC Health Services Research 2010 10(Suppl 2): A13.
Submit your next manuscript to BioMed Central and take full advantage of:

- Convenient online submission

- Thorough peer review

- No space constraints or color figure charges

- Immediate publication on acceptance

- Inclusion in PubMed, CAS, Scopus and Google Scholar

- Research which is freely available for redistribution

Submit your manuscript at www.biomedcentral.com/submit 